\title{
The immunological profile of maternal obesity at 28 weeks of gestation underpins common negative pregnancy outcomes
}

\author{
April Rees ${ }^{1}$, Oliver Richards ${ }^{1}$, Nick Jones ${ }^{1}$, and Catherine Thornton ${ }^{2}$ \\ ${ }^{1}$ Swansea University \\ ${ }^{2}$ Swansea University, Institute of Life Sciences
}

September 24, 2021

\begin{abstract}
Healthy pregnancy is accompanied by various immunological and metabolic adaptations. Maternal obesity has been implicated in adverse pregnancy outcomes such as miscarriage, preeclampsia, and gestational diabetes mellitus (GDM), while posing a risk to the neonate. There is a lack of knowledge surrounding obesity and the maternal immune system. The objective of this study was to consider if immunological changes in pregnancy are sabotaged by maternal obesity. Peripheral blood was collected from fasted GDM-negative pregnant women at 26-28 weeks of gestation. Analysis was done using immunoassay, flow cytometry, bioenergetics analysis and cell culture. The plasma profile was significantly altered with increasing BMI, specifically leptin $(\mathrm{r}=0.7635)$, MCP-1 $(\mathrm{r}=0.3024)$ and IL-6 $(\mathrm{r}=0.4985)$. Circulating leukocyte populations were also affected with changes in the relative abundance of intermediate monocytes $(\mathrm{r}=-0.2394)$, CD4:CD8 $\mathrm{T}$ cell ratios $(\mathrm{r}=0.2789)$, and NKT cells $(\mathrm{r}=-0.2842)$. Monocytes analysed in more detail revealed elevated CCR2 expression and decreased mitochondrial content. However, LPS-stimulated cytokine production and bioenergetic profile of MNCs was not affected by maternal BMI. The Th profile skews towards Th17 with increasing BMI; Th2 ( $\mathrm{r}=-0.3202)$ and Th9 $(\mathrm{r}=-0.3205)$ cells were diminished in maternal obesity, and CytoStimTM-stimulation exacerbates IL-6 ( $\mathrm{r}=0.4166)$, IL-17A ( $\mathrm{r}=0.2753)$, IL-17F $(\mathrm{r}=0.2973)$ and IL-22 (r=0.2257) production with BMI, while decreasing IL-4 ( $\mathrm{r}=-0.2806)$. Maternal obesity during pregnancy creates an inflammatory microenvironment. Successful pregnancy requires Th2-biased responses yet increasing maternal BMI favours a Th17 response that could be detrimental to pregnancy. Further research should investigate key populations of cells identified here to further understand the immunological challenges that beset pregnant women with obesity.
\end{abstract}

\section{Hosted file}

28 week gestation obesity.docx available at https://authorea.com/users/434962/articles/ 538459-the-immunological-profile-of-maternal-obesity-at-28-weeks-of-gestation-underpinscommon-negative-pregnancy-outcomes

\section{Hosted file}

28_week_gestation_obesity.pdf available at https://authorea.com/users/434962/articles/538459the-immunological-profile-of-maternal-obesity-at-28-weeks-of-gestation-underpins-commonnegative-pregnancy-outcomes 